R4178

\title{
The Role of Sulfide in Reticulophagy through the Regulation of ATG18a by Persulfidation
}

Angeles Aroca, Diane C. Bassham

Iowa State University

Autophagy is a major catabolic process in eukaryotic cells to degrade dysfunctional or unnecessary cellular components. It has conserved functions in development, cellular homeostasis, and stress responses from yeast to plants and mammals. Autophagy is implicated in cancer, liver disease, neurodegeneration, cardiac disease, pathogen infection, among other pathological symptoms in mammals, but in plants, it is critically important in the plant life, including seedling establishment, plant development, stress resistance, metabolism and reproduction. Autophagy also contributes to intracellular homeostasis in cells by selectively degrading aggregated proteins, damaged mitochondria, ribosomes, toxic macromolecules, excess peroxisomes, and pathogens to prevent toxicity. This selective autophagy is mediated by the binding of adaptor proteins, which link a cargo targeted for degradation to the autophagosome machinery. An increasing number of targets for selective autophagy under different stress conditions have emerged in recent years, but the underlying mechanisms of their regulation are still so far unknown. Hydrogen sulfide $\left(\mathrm{H}_{2} \mathrm{~S}\right)$ is an endogenously generated gaseous signaling molecule, which has been recently implicated in autophagy regulation in both plants and mammals through persulfidation of specific targets. In previous works, it has been suggested that persulfidation may be the molecular mechanism through which sulfide regulates autophagy in plant cells. ATG18a is a core autophagy component that is required for bulk autophagy and is required for reticulophagy during ER stress and RNA interference (RNAi)-ATG18a transgenic plants showed an autophagy-defective phenotype. Thus, persulfidation of ATG18a may regulate ER stress-induced autophagy in Arabidopsis, in a similar way as other posttranslational modifications (PTMs) regulate the ability of the ATG proteins to interact with a number of autophagic regulators. In this research, we have revealed the role of sulfide in ER stress as a negative regulator of autophagy in plants. We demonstrate that persulfidation in ATG18a is sulfide-concentration-dependent using a dimedone switch method with Cy5 as reporting molecule for persulfide groups. We also show that sulfide regulates ATG18a phospholipid-binding activity by reversible persulfidation in a specific residue. Our findings demonstrate that sulfide significantly activates AtATG18a binding capacity to specific phospholipids in a reversible manner.

\section{Support or Funding Information}

This project has received funding from the European Union's Horizon 2020 research and innovation program under the Marie Skłodowska-Curie Individual Fellowship grant agreement No 834120 .

Print Close

